\title{
The Upper Ascending Reticular Activating System between Intralaminar Thalamic Nuclei and Cerebral Cortex in the Human Brain
}

\author{
Sungho Jang, Soyoung Kwak \\ Department of Physical Medicine \& Rehabilitation, College of Medicine, Yeungnam University, Daegu, Korea
}

\begin{abstract}
Purpose: The ascending reticular activating system (ARAS) is responsible for regulation of consciousness. In this study, using diffusion tensor imaging (DTI), we attempted to reconstruct the thalamocortical projections between the intralaminar thalamic nuclei and the frontoparietal cortex in normal subjects.

Methods: DTI data were acquired in 24 healthy subjects and eight kinds of thalamocortical projections were reconstructed: the seed region of interest (ROI) - the intralaminar thalamic nuclei and the eight target ROls - the medial prefrontal cortex, dorsolateral prefrontal cortex, ventrolateral prefrontal cortex, orbitofrontal cortex, premotor cortex, primary motor cortex, primary somatosensory cortex, and posterior parietal cortex.

Results: The eight thalamocortical projections were reconstructed in each hemisphere and the pathways were visualized: projections to the prefrontal cortex ascended through the anterior limb and genu of the internal capsule and anterior corona radiata. Projections to the premotor cortex passed through the genu and posterior limb of the internal capsule and middle corona radiata; in contrast, projections to the primary motor cortex, primary somatosensory cortex, and posterior parietal cortex ascended through the posterior limb of the internal capsule. No significant difference in fractional anisotropy, mean diffusivity, and fiber volume of all reconstructed thalamocortical projections was observed between the right and left hemispheres ( $p>0.05)$.

Conclusion: We reconstructed the thalamocortical projections between the intralaminar thalamic nuclei and the frontoparietal cortex in normal subjects. We believe that our findings would be useful to clinicians involved in the care of patients with impaired consciousness and for researchers in studies of the ARAS.
\end{abstract}

Keywords: Ascending reticular activating system, Diffusion tensor imaging, Thalamus, Intralaminar nuclei, Consciousness

\section{INTRODUCTION}

The ascending reticular activating system (ARAS) is responsible for the regulation of consciousness, which consists of arousal and awareness. ${ }^{1-4}$ The ARAS originates mainly from the reticular formation of the brainstem and exerts its influence on the cerebral cortex via two main routes: 1) dorsal pathway; via nonspecific thalamic nuclei, and 2) ventral pathway; via cholinergic nuclei in the basal forebrain. ${ }^{1-3,5}$ The nonspecific thalamic nuclei, including the intralaminar nuclei, midline nuclei, and reticular nuclei constitute the thalamocortical projections as the classical ARAS. ${ }^{3}$

Received Apr 26, 2017 Revised Jun 7, 2017

Accepted Jun 7, 2017

Corresponding author Soyoung Kwak

E-mail soyoung.kwak@daum.net
Many patients with various brain pathologies such as hypoxic ischemic brain injury, traumatic brain injury, and stroke, have been reported to suffer from impaired consciousness, which can be a significant burden to the patient's family and society. ${ }^{2,67}$ Although accurate diagnosis of the presence and severity of injury of the ARAS in patients with impaired consciousness is necessary, accurate identification and estimation of the ARAS in the human brain was problematic prior to the development of diffusion tensor imaging (DTI). DTI enables the reconstruction and evaluation of some important portions of the ARAS. ${ }^{8-10}$ Many studies have demonstrated the usefulness of DTI for evaluating lesions in patients with im-

Copylight (C)2017 The Korea Society of Physical Therapy

This is an Open Access article distribute under the terms of the Creative Commons Attribution Non-commercial License (Http:// creativecommons.org/license/by-nc/4.o.) which permits unrestricted non-commercial use, distribution, and reproduction in any medium, provided the original work is properly cited. 
paired consciousness. ${ }^{911-18}$ However, little is known about the thalamocortical projections between nonspecific thalamic nuclei and the cerebral cortex in the human brain. ${ }^{9}$

In the current study, using DTI tractography, we attempted to reconstruct the thalamocortical projections between the intralaminar thalamic nuclei and the frontoparietal cortex in normal subjects.

\section{METHODS}

\section{Subjects}

Twenty four healthy subjects (13 males, 11 females, mean age: 34.65 \pm 12.53 years, range: 20 to 50 ) with no history of neurologic disease were recruited for this study. All subjects participated in this study as volunteers and provided written consent before undergoing DTI scanning. The study was approved by the institutional review board of a university hospital.

\section{Diffusion tensor image}

Acquisition of DTI data was performed using a 6-channel head coil on a 1.5 T Philips gyroscan intera (Philips, Best, The Netherlands) and single-shot echo-planar imaging. For each of the 32 non-collinear diffusion sensitizing gradients, we acquired 67 contiguous slices parallel to the anterior commissure-posterior commissure line. Imaging parameters were as follows: acquisition matrix $=96 \times 96$, reconstructed matrix $=192 \times 192$, field of view $=240 \times 240 \mathrm{~mm}^{2}, \mathrm{TR}=10,726 \mathrm{~ms}$, $\mathrm{TE}=76 \mathrm{~ms}$, parallel imaging reduction factor $(\mathrm{SENSE}$ factor $)=2, \mathrm{EPI}$
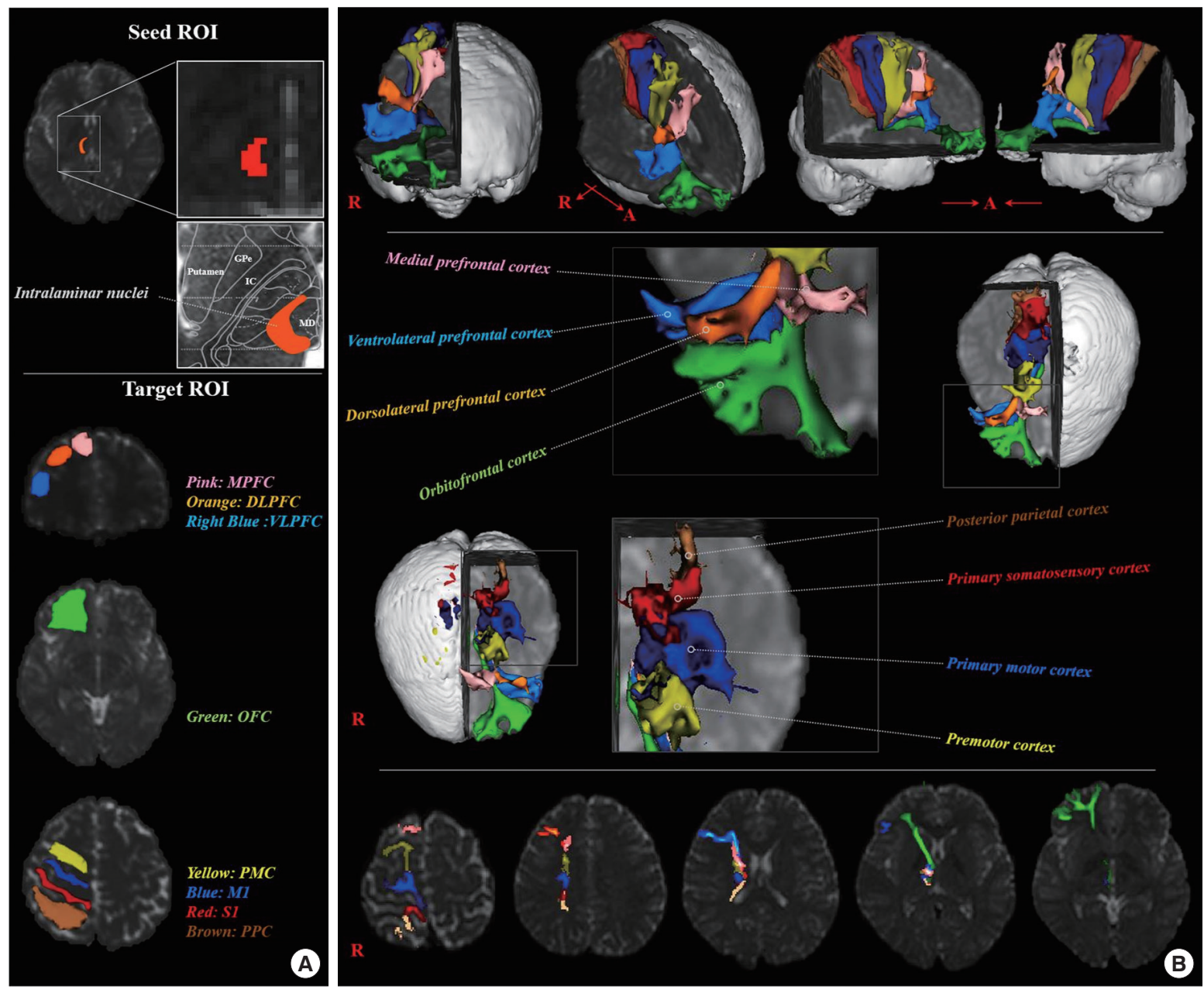

Figure 1. (A) Seed region of interest (ROI) is placed on the intralaminar nuclei of the thalamus at the level of the inter-commissural plane. Target $\mathrm{ROI}$ is placed on the medial prefrontal cortex (MPFC), dorsolateral prefrontal cortex (DLPFC), ventrolateral prefrontal cortex (VLPFC), orbitoprefrontal cortex (OFC), premotor cortex (PMC), primary motor cortex (M1), primary somatosensory cortex (S1), and posterior parietal cortex (PPC), respectively. (B) The neural pathways of thalamocortical projections between the intralaminar thalamic nuclei and each target ROI are shown at each brain level in a normal subject (a 24-year-old male). 
factor $=49, \mathrm{~b}=1,000 \mathrm{~s} / \mathrm{mm}^{2}, \mathrm{NEX}=1$, and a slice thickness of $2.5 \mathrm{~mm}$ with no gap (acquired voxel size $1.3 \times 1.3 \times 2.5 \mathrm{~mm}^{3}$ ).

\section{Probabilistic fiber tracking}

Analysis of diffusion-weighted imaging data was performed using software from the Oxford centre for functional magnetic resonance imaging of the brain (FMRIB) software library (FSL v5.0, www.fmrib.ox.ac.uk/fsl). Affine multi-scale two-dimensional registration was used for correction of head motion and eddy current-induced image distortion. Fiber tracking was performed using a probabilistic tractography method based on a multifiber model using tractography routines implemented in FMRIB Diffusion (5,000 streamline samples, $0.5 \mathrm{~mm}$ step lengths, curvature thresholds $=0.2){ }^{19}$

In each subject, the seed region of interest (ROI) was placed on the intralaminar thalamic nuclei at the level of the inter-commissural plane between the anterior and posterior commissures. ${ }^{10,20,21}$ The target ROIs were placed for each cortical area as follows, respectively: the medial prefrontal cortex (MPFC) - Brodmann area (BA) 32, dorsolateral prefrontal cortex (DLPFC) - BA 8, 9, and 46, ventrolateral prefrontal cortex (VLPFC) - BA 44, 45, and 47, orbitofrontal cortex (OFC) - BA 10, 11, 12, and 47, premotor cortex (PMC) - BA 6, primary motor cortex (M1) - BA 4, primary somatosensory cortex (S1) - BA 1, 2, and 3, and posterior parietal cortex (PPC) - BA 5 and $7 .^{22-25}$ We drew the target ROIs manually on the coronal image for the MPFC, DLPFC, and VLPFC and on the axial image for the OFC, PMC, M1, S1, and PPC (Figure 1A). Boundaries of each target ROI was set as follows: BA 6-the anterior boundary: the line drawn through the anterior commissure perpendicular to the anterior commissure-posterior commissure line, the posterior boundary: the precentral sulcus, MPFC- medial boundary: the midline between the right and left hemisphere, lateral boundary: superior frontal sulcus, DLPFC- medial boundary: superior frontal sulcus; lateral boundary: inferior frontal sulcus, VLPFC - medial boundary: inferior frontal sulcus, lateral boundary: Sylvian fissure, OFC the anterior boundary: rostral part of the central orbital region, the posterior boundary: opercular part of inferior frontal gyrus, M1-the anterior boundary: the precentral sulcus, the posterior boundary: the central sulcus, S1-the anterior boundary: the central sulcus, the posterior boundary: the postcentral sulcus; PPC- the anterior boundary: the postcentral sulcus, the posterior boundary: the superior parietal lobule. ${ }^{22-24}$ The thalamocortical projections between the intralaminar thalamic nuclei and the frontoparietal cortex were determined by selection of fibers passing through the seed and each target ROI. There were 5,000 samples generated from the seed voxel, and the results were visualized at the threshold of 1 streamline through each voxel for analysis. Values of fractional anisotropy (FA), mean diffusivity (MD), and fiber volume of each thalamocortical projection were measured.

\section{Statistical analysis}

SPSS software (v.15.0, SPSS, Chicago, IL) was used for data analysis. Independent t-test was used in determining the significance of difference in DTI parameters of reconstructed thalamocortical projections between right and left hemispheres. Statistical significance was accepted for $\mathrm{p}$-values of $<0.05$.

\section{RESULTS}

The eight reconstructed thalamocortical projections between the intralaminar thalamic nuclei and the frontoparietal cortex are shown in Figure 1. Although some individual variations were observed, the eight thalamocortical projections between the intralaminar thalamic nuclei and each frontoparietal cortex were reconstructed in all subjects, and the main course of the thalamocortical projections to the prefrontal cortex was as follows (Figure 2A): the thalamocortical projections to the MPFC and DLPFC ascended through the anterior limb (100\%) and genu of the upper internal capsule (100\%), the anterior portion of lower corona radiata (MPFC = anterior: 95\%, middle: 65\%, DLPFC: anterior: 95\%, middle: $45 \%$ ), anterior portion of upper corona radiata $(\mathrm{MPFC}=$ anterior: 100\%, middle: 20\%, DLPFC: anterior: 100\%, middle: 5\%), and the anteromedial and anterolateral centrum semiovale, and then terminated in the MPFC and DLPFC, respectively. The thalamocortical projections to the VLPFC also ascended through the anterior limb $(100 \%)$ and genu (100\%) of the upper internal capsule, the anterior and middle portion of corona radiata around the anterior horn of the lateral ventricle (lower = anterior: $100 \%$, middle: $55.0 \%$, upper = anterior: $85.0 \%$, middle: $0 \%$ ), and then terminated in the VLPFC. The thalamocortical projections to the OFC passed horizontally through the anterior limb (100\%) and genu (100\%) of the lower internal capsule, anterior and middle portion of lower corona radiata (anterior: 90\%, middle: 45.0\%) and terminated in the OFC. 


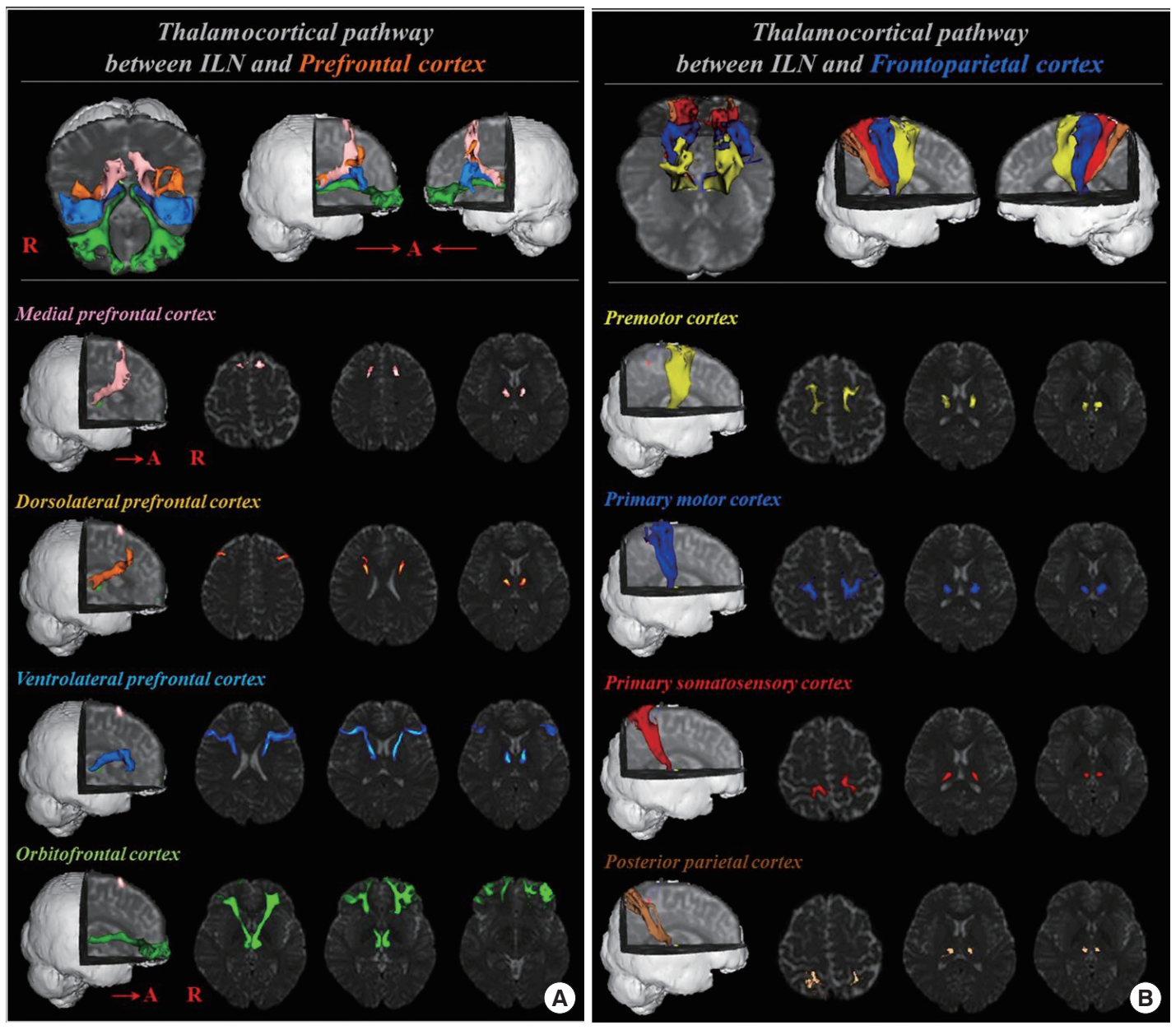

Figure 2. (A) The neural pathways of thalamocortical projections between the intralaminar nuclei of the thalamus and four prefrontal cortices (medial prefrontal cortex, dorsolateral prefrontal cortex, ventrolateral prefrontal cortex, orbitofrontal cortex), (B) and between the intralaminar nuclei of the thalamus and four frontoparietal cortices (premotor cortex, primary motor cortex, primary somatosensory cortex, posterior parietal cortex) are shown at each brain level in a normal subject (a 24-year-old male).

The thalamocortical projections to the PMC ascended through the genu $(80 \%)$ and posterior limb $(100 \%)$ of the upper internal capsule, the corona radiata around the middle portion of the lateral ventricle $($ lower $=$ middle: $100 \%$, posterior: $5 \%$, upper $=$ middle: $100 \%$, posterior: $20 \%$ ) and the anterior centrum semiovale, and then terminated in the PMC. The thalamocortical projections to the M1 ascended through the genu (30\%) and posterior limb (100\%) of the upper internal capsule, the corona radiata around the middle portion of the lateral ventricle (lower = middle: $80 \%$, posterior: $75 \%$, upper $=$ middle: $70 \%$, posterior: $90 \%$ ) and M1 through the middle centrum semiovale, and then terminated in the M1. By contrast, the thalamocortical projections to the M1, S1, and PPC ascended through the posterior limb (100\%) of the upper internal capsule and the middle and posterior portion of corona radiata (lower = middle: $40 \%$, posterior: $100 \%$; upper $=$ middle: $35 \%$, posterior: $100 \%$ ), and then terminated in the M1 through the middle centrum semiovale and in the S1 and PPC through the posterior centrum semiovale.

A summary of the mean values for FA, MD, and fiber volume of the reconstructed thalamocortical projections between the intralaminar thalamic nuclei and the frontoparietal cortex is shown in Table 1. No significant differences in FA, MD, and fiber volume of all reconstructed thalamocortical projection fibers were observed between right and left hemispheres ( $p>0.05$ ).

\section{DISCUSSION}

In the current study, using DTI tractography, we reconstructed the thalamocortical projections between the intralaminar thalamic nuclei and the frontoparietal cortex in 24 normal subjects. ROIs were placed as follows: the seed ROI - the intralaminar thalamic nuclei at 
Table 1. Diffusion tensor imaging parameters of thalamocortical projections between the intralaminar thalamic nuclei and the frontoparietal cortex

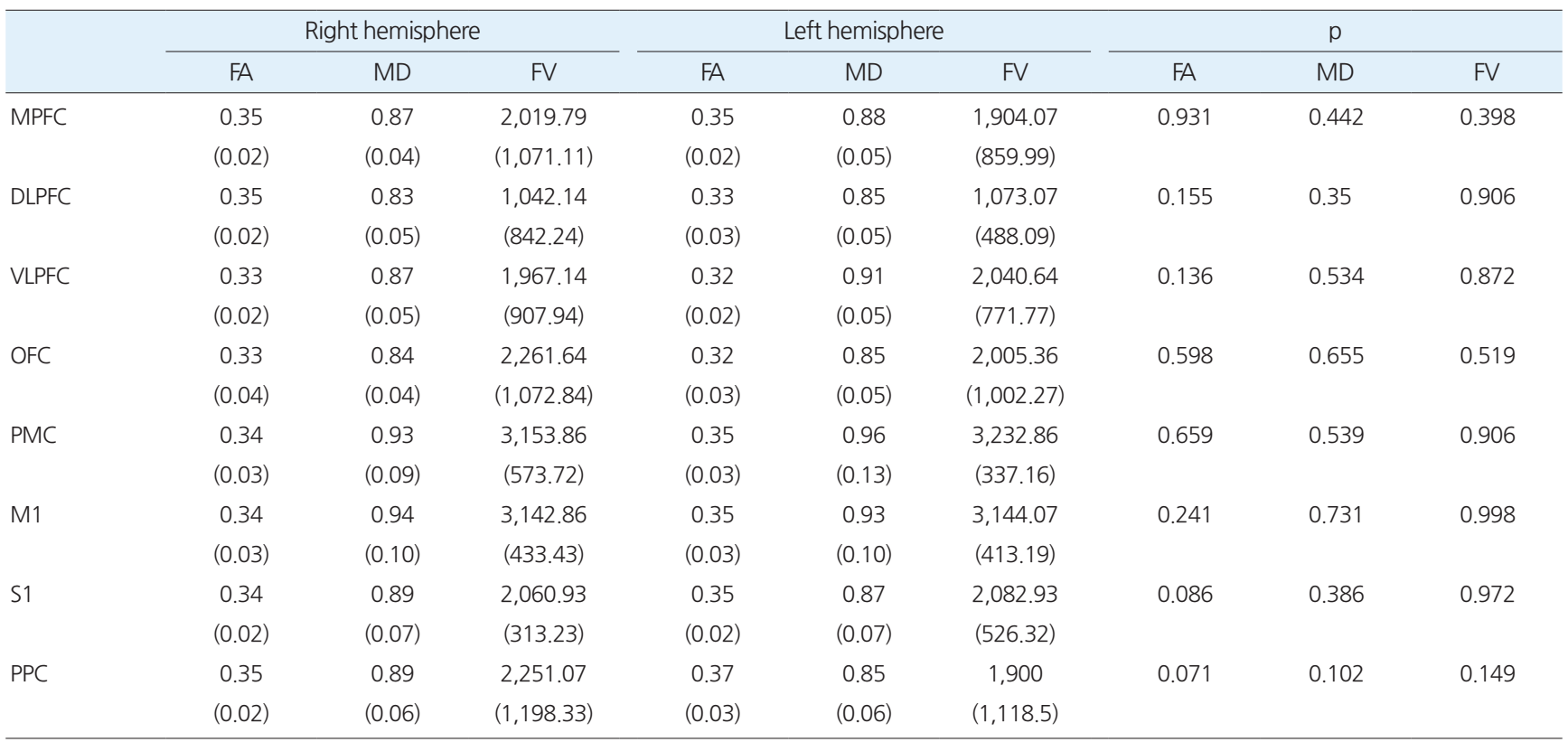

Values are expressed as mean (standard deviation).

FA: fractional anisotropy, MD: mean diffusivity $M D \times 10^{-3}\left(\mathrm{~mm}^{2} / \mathrm{s}\right)$, FV: fiber volume, MPFC: medial prefrontal cortex, DLPFC: dorsolateral prefrontal cortex, VLPFC: ventrolateral prefrontal cortex, OFC: orbitofrontal cortex, PMC: premotor cortex, M1: primary motor cortex, S1: primary somatosensory cortex, PPC: posterior parietal cortex. Independent t-test was used for comparison of the difference of diffusion tensor imaging parameters of thalamocortical connection fibers between right and left hemisphere.

the level of the inter-commissural line and the target ROIs - the eight cerebral areas of the frontoparietal cortex. Our findings were as follows: 1) the eight thalamocortical projections were reconstructed in both hemispheres and the pathways between the intralaminar thalamic nuclei and the frontoparietal cortex were visualized, the projections to the prefrontal cortex (MPFC, DLPFC, and VLPFC) ascended through the anterior limb and genu of the internal capsule, and anterior corona radiata. The projections to the PMC passed through the genu and posterior limb of the internal capsule, and middle corona radiata, in contrast, the projections to the M1, S1, and PPC ascended through the posterior limb of the internal capsule, and 2) DTI parameters, including FA, MD, and fiber volume of each thalamocortical projection were measured; no significant difference was observed between right and left hemispheres.

Since introduction of DTI, many studies have reported on the clinical usefulness of DTI by estimating some areas of the ARAS in patients with impaired consciousness. ${ }^{8,912,13,15-18}$ Most of these studies have measured DTI parameters using the ROI method at nonspecific areas of the brain without three-dimensional reconstruction of the ARAS. ${ }^{12,13,15-18}$ Regarding the thalamocortical projections between nonspecific thalamic nuclei and the cerebral cortex as a part of the ARAS, to the best of our knowledge, Eldow et al. ${ }^{9}$ per- formed the first three-dimensional reconstruction in a normal subject and a patient with coma following traumatic brain injury. They placed ROIs in the intralaminar thalamic nuclei (central lateral nucleus, centromedian nucleus, parafascicular nucleus, and reticular nucleus) for analysis of thalamocortical connectivity and non-relevant fiber tracts were eliminated by subtracting all thalamic fiber tracts connected to the brainstem, according to their results, the connectivity was partially disrupted in the patient compared with the control subject. By contrast, we reconstructed the thalamocortical projections by placing ROIs in the intralaminar thalamic nuclei and eight cerebral cortical areas of the frontoparietal cortex in 24 normal subjects.

In conclusion, using DTI tractography, we reconstructed the thalamocortical projections between the intralaminar thalamic nuclei and the frontoparietal cortex in normal subjects. We believe that the reconstruction methodology used for these projections and the results of this study would be useful to clinicians involved in the care of patients with impaired consciousness and researchers in the studies of the ARAS. However, the limitations of this study should be considered. First, we did not perform an analysis of the thalamocortical projections to the occipito-temporal cortex, which is a component of the ARAS., ${ }^{3,426}$ In addition, we did not include other tha- 
lamic and brainstem nuclei that are also involved in the ARAS in our analysis. Therefore, conduct of further studies on these topics should be encouraged. Second, DTI is a powerful anatomic imaging tool, which can demonstrate gross fiber architecture; however, due to crossing fiber or partial volume effect, DTI can produce both false positive and negative results throughout the white matter of the brain. ${ }^{27-29}$

\section{ACKNOWLEDGEMENTS}

This work was supported by the National Research Foundation (NRF) of Korea Grant funded by the Korean Government (MSIP) (2015R1A2A2A01004073).

\section{REFERENCES}

1. Paus T. Functional anatomy of arousal and attention systems in the human brain. Prog Brain Res. 2000;126:65-77.

2. Zeman A. Consciousness. Brain. 2001;124:1263-89.

3. Daube JR. Medical neurosciences: an approach to anatomy, pathology, and physiology by systems and levels. 2nd ed. Boston, Little, Brown and Co., 1986:450.

4. Schiff ND. Central thalamic contributions to arousal regulation and neurological disorders of consciousness. Ann N Y Acad Sci. 2008; 1129:105-18.

5. Afifi AK, Bergman RA. Functional neuroanatomy : text and atlas. 2nd ed. New York, Lange Medical Books/McGraw-Hill, 2005:494.

6. Gosseries O, Bruno MA, Chatelle C et al. Disorders of consciousness: what's in a name? NeuroRehabilitation. 2011;28(1):3-14.

7. Pisa FE, Biasutti E, Drigo D et al. The prevalence of vegetative and minimally conscious states: a systematic review and methodological appraisal. J Head Trauma Rehabil. 2014;29(4):E23-30.

8. Edlow BL, Takahashi E, Wu O et al. Neuroanatomic connectivity of the human ascending arousal system critical to consciousness and its disorders. J Neuropathol Exp Neurol. 2012;71(6):531-46.

9. Edlow BL, Haynes RL, Takahashi E et al. Disconnection of the ascending arousal system in traumatic coma. J Neuropathol Exp Neurol. 2013; 72(6):505-23.

10. Yeo SS, Chang PH, Jang SH. The ascending reticular activating system from pontine reticular formation to the thalamus in the human brain. Front Hum Neurosci. 2013;7:416.

11. Voss HU, Uluc AM, Dyke JP et al. Possible axonal regrowth in late recovery from the minimally conscious state. J Clin Invest. 2006;116(7): 2005-11.

12. Perlbarg V, Puybasset L, Tollard E et al. Relation between brain lesion location and clinical outcome in patients with severe traumatic brain inju- ry: a diffusion tensor imaging study using voxel-based approaches. Hum Brain Mapp. 2009;30(12):3924-33.

13. Tollard E, Galanaud D, Perlbarg V et al. Experience of diffusion tensor imaging and $1 \mathrm{H}$ spectroscopy for outcome prediction in severe traumatic brain injury: preliminary results. Crit Care Med. 2009;37(4):144855 .

14. Tshibanda L, Vanhaudenhuyse A, Galanaud D et al. Magnetic resonance spectroscopy and diffusion tensor imaging in coma survivors: promises and pitfalls. Prog Brain Res. 2009;177:215-29.

15. Fernandez-Espejo D, Junque C, Cruse D et al. Combination of diffusion tensor and functional magnetic resonance imaging during recovery from the vegetative state. BMC Neurol. 2010;10:77.

16. Newcombe VF, Williams GB, Scoffings D et al. Aetiological differences in neuroanatomy of the vegetative state: insights from diffusion tensor imaging and functional implications. J Neurol Neurosurg Psychiatry. 2010;81(5):552-61.

17. Fernandez-Espejo D, Bekinschtein T, Monti MM et al. Diffusion weighted imaging distinguishes the vegetative state from the minimally conscious state. NeuroImage. 2011;54(1):103-12.

18. Luyt CE, Galanaud D, Perlbarg V et al. Diffusion tensor imaging to predict long-term outcome after cardiac arrest: a bicentric pilot study. Anesthesiology. 2012;117(6):1311-21.

19. Smith SM, Jenkinson M, Woolrich MW et al. Advances in functional and structural MR image analysis and implementation as FSL. Neuroimage. 2004;23(Suppl 1):S208-19.

20. Morel A, Magnin M, Jeanmonod D. Multiarchitectonic and stereotactic atlas of the human thalamus. J Comp Neurol. 1997;387(4):588-630.

21. Morel A. Stereotactic atlas of the human thalamus and basal ganglia. New York, Informa Healthcare, 2007:149.

22. Johansen-Berg H, Behrens TE, Sillery E et al. Functional-anatomical validation and individual variation of diffusion tractography-based segmentation of the human thalamus. Cereb Cortex. 2005;15(1):31-9.

23. Klein JC, Rushworth MF, Behrens TE et al. Topography of connections between human prefrontal cortex and mediodorsal thalamus studied with diffusion tractography. NeuroImage. 2010;51(2):555-64.

24. Kringelbach ML. The human orbitofrontal cortex: linking reward to hedonic experience. Nat Rev Neurosci. 2005;6(9):691-702.

25. Brodmann K, Gary LJ. Brodmann's localization in the cerebral cortex: the principles of comparative localisation in the cerebral cortex based on cytoarchitectonics. New York, Springer, 2006:298.

26. Schiff ND. Recovery of consciousness after brain injury: a mesocircuit hypothesis. Trends Neurosci. 2010;33(1):1-9.

27. Lee SK, Kim DI, Kim J et al. Diffusion-tensor MR imaging and fiber tractography: a new method of describing aberrant fiber connections in developmental CNS anomalies. Radiographics. 2005;25(1):53-65.

28. Parker GJ, Alexander DC. Probabilistic anatomical connectivity derived from the microscopic persistent angular structure of cerebral tissue. Philos Trans R Soc Lond B Biol Sci. 2005;360(1457):893-902.

29. Yamada K, Sakai K, Akazawa K et al. MR tractography: a review of its clinical applications. Magn Reson Med Sci. 2009;8(4):165-74. 